\title{
Control of Fibrinogen Biosynthesis: Role of the FFA/Albumin Ratio
}

\author{
Laurence Pilgeram
}

Published online: 10 April 2010

(C) The Author(s) 2010. This article is published with open access at Springerlink.com

\begin{abstract}
The rate of biosynthesis and turnover of the plasma protein fibrinogen is a marker of metabolic signaling in aging and disease. The rate in the young normal human subject of $0.260 \mathrm{mg} / \mathrm{ml} / 24 \mathrm{~h}$ increases to 0.378 in older normal subjects and to 0.466 in age matched coronary thrombosis patients measured by endogenous labeling of fibrinogen with L-glutamic acid-C14. The increased rate of fibrinogen turnover has been traced to generation of fibrin by labeling the polymers with glycine C14 ethyl esters in the presence of activated fibrin stabilizing factor. Circulating fibrin increased $520 \%$ above normal in ischemic thrombotic cerebrovascular disease. Long chain saturated free fatty acids (FFA) exercise not only primary control over incorporation of $\mathrm{Cl} 4$ labeled amino acids into the fibrinogen structure but also activate the cascade sequence of reactions which convert fibrinogen into occlusive fibrin polymers. FFA are normally bound and transported by plasma albumin to mitochondrial sites of energy metabolism. Albumin synthesis declines with aging. This decline is associated with increased plasma levels of FFA resulting in an increase in the plasma FFA/albumin ratio. Correction of this ratio in vitro by restoration of a normal FFA/albumin ratio restores a normal level of fibrinogen synthesis by human hepatocytes.
\end{abstract}

Keywords Fibrinogen - Fatty acids · Fibrin - Albumin . Thrombosis $\cdot$ Risk factor $\cdot$ Metabolic signaling

L. Pilgeram $(\bowtie)$

Department of Molecular, Cellular, and Developmental Biology,

University of California, Santa Barbara, CA, USA

e-mail: eric.farr@springer.com

\section{Introduction}

Increased plasma fibrinogen in coronary heart disease has been known since 1959 (Schram and Pilgeram 1959; Pilgeram 1961, 1993) and identified as a risk factor for future cardiovascular events in prospective long term studies of apparently healthy subjects (Stone and Thorp 1985; Meade et al. 1986; Kannel et al. 1987; Yarnell et al. 1991; Burr et al. 1992) and in patients with coronary heart disease (Heinrich et al. 1994; Benderly et al. 1996). Deteriorating prognosis in patients with unstable coronary heart disease is associated with increased fibrinogen levels (Toss et al. 1997). Early signs of occlusive vascular disease in patients who are asymptomatic have been associated with increased fibrinogen levels (Levenson et al. 1995; Tracy et al. 1995). Monitoring of the plasma level of fibrinogen in high risk patients, who had experienced previous ischemic events, found the plasma level of fibrinogen to progressively increase prior to the clinically overt ictus of coronary thrombosis and thrombotic stroke (Pilgeram 1984).

These reports present a crucial question on the relevance of this increase to thrombogenesis, coronary heart disease, thrombotic stroke and potential preventive therapy. Was the increased plasma level of fibrinogen due to increased synthesis and turnover into occlusive fibrin or due to depressed utilization of fibrinogen as a physiological corrective action? To answer this question, a technology was developed based on endogenous labeling of fibrinogen with L-glutamic acid-C14 which enabled measuring the in vivo rate of biosynthesis and turnover of fibrinogen. This research demonstrated the increase in the plasma level of fibrinogen to be due to increased synthesis and turnover. The rate in the young normal human subject of $0.260 \mathrm{mg} / \mathrm{ml} / 24 \mathrm{~h}$ increased to 0.378 in older normal subjects and to 0.466 in age matched patients 
who had recovered from verified myocardial infarction (Pilgeram 1968).

This increased rate of synthesis and turnover of fibrinogen was traced to generation of fibrin by development of a technology which labeled circulating fibrin molecules with glycine-C14 ethyl esters in the presence of activated fibrin stabilizing factor. Circulating fibrin was found to increase $520 \%(P<0.001)$ above normal in patients who had incurred and recovered from ischemic thrombotic cerebrovascular disease (Pilgeram et al. 1973; Pilgeram 1973).

A screening study on the effect of multiple factors on the in vitro rate of synthesis of fibrinogen in hepatocytes, measured by the incorporation of C14 labeled amino acids into the protein structure, identified long chain saturated fatty acids as the primary factor (Pilgeram and Pickart 1968). Di-nitro phenol, a specific inhibitor of protein synthesis, prevented this action of fatty acids thus establishing specificity at the level of protein synthesis. In designing a study aimed at determining if dysfunctional fatty acid metabolism was responsible for increased synthesis of fibrinogen in thrombosis, focus was aimed at the plasma protein albumin because albumin had been identified as the obligatory co-factor which accepts free fatty acids (FFA) released during corrective clearing of alimentary lipemia by lipoprotein lipase in patients who had experienced coronary thrombosis (Pilgeram 1958; Pilgeram et al. 1964). Although the plasma level of FFA is influenced by a number of factors, including dietary and mobilization from adipose tissue fat stores, the primary factor was considered to be the degree to which FFA is bound and transported by albumin. Focus was therefore directed to determining whether the plasma level of FFA could be correlated with thrombosis and the plasma level of albumin. The objective was to determine whether the physical chemical binding coefficient between albumin and FFA, measured by the FFA/albumin molar ratio, regulated the rate of fibrinogen synthesis. A regulatory function could identify deficient albumin synthesis as the cause of increased synthesis and turnover of fibrinogen into occlusive fibrin of coronary thrombosis and thrombotic stroke.

\section{Methods}

\section{Plasma Specimens}

Human plasma samples were obtained from 53 male Caucasian subjects between 20 and 70 years of age who were diagnosed as clinically normal. Pathological plasma samples were obtained from 29 male Caucasian patients between 30 and 70 years of age with diagnosis of having recovered from myocardial infarction or thrombotic stroke with no evidence of other disease. Plasma was assayed for albumin and non esterified or free fatty acids (FFA). Blood was withdrawn after an overnight fast of $12 \mathrm{~h}$ or more. The minimum requirements for accepting a patient as having coronary heart disease or thrombotic stroke were (a) a clinical and laboratory history of myocardial infarction or thrombotic stroke, and (b) electrocardiographic or neurological and electroencephalographic change characteristic of an infarction or thrombotic stroke. All donors were free of drug therapy for a period of 6 weeks prior to blood sampling. Anticoagulant consisted of $0.75 \mathrm{ml}$ of $6.4 \%$ trisodium citrate per $15 \mathrm{ml}$ of blood.

\section{Albumin Assay-Electrophoretic}

Isolation of plasma albumin was processed in the Durrum paper electrophoresis cell. The isolated albumin band was stained, eluted and quantitated spectrophotometrically. The method is described in the Beckman Technical Bulletin No. TB6O5OA, Procedure B. Each sample was run in triplicate.

\section{Free Fatty Acid (FFA) Assay}

FFA was extracted from plasma, titrated, and measured according to the method of Dole and Meinertz (1960) and confirmed by gas chromatography (Beckman GC-4) on diethylene succinate using reference standards.

\section{Measure of Fibrinogen Synthesis}

Methods for isolation and measure of plasma fibrinogen and the specificity of these methods have been published (Pilgeram 1961, 1968; Pilgeram and Pickart 1967).

\section{FFA Extracted Albumin}

Human albumin, Cohn's Fraction V (Bayer, Berkeley, $\mathrm{CA}), 5 \%$ in $0.85 \mathrm{~g} \%$ saline, was precipitated in an equal volume of $10 \%$ trichloracetic acid. The precipitate was centrifuged $(1,000 \mathrm{~g}$ for $10 \mathrm{~min})$, extracted into $95 \%$ ethanol (ethanol volume: $10 \times$ original albumin volume), then centrifuged $5,000 \mathrm{~g}$ for $30 \mathrm{~min}$. The supernatant ethanol solution is poured slowly into $0^{\circ} \mathrm{C}$ ether, then dried under vacuum. The dried albumin was re-dissolved in distilled water, dialyzed, and then lyophilized. This procedure reduced the molar ratio of FFA/albumin from 1.16 to 0.13 measured by gas chromatography. Albumin electrophoretic pattern and resolution were not altered by this procedure.

In Vitro Hepatocyte Synthesis of Fibrinogen

Human hepatocytes, $100 \mathrm{mg}$, were incubated for $2 \mathrm{~h}$ at $37^{\circ}$ $\mathrm{C}$ in $2 \mathrm{ml}$ of human plasma and 5 micro Curies of glycine- 
C14 in a shaking water bath. Heparin was added as an anti coagulant at the concentration of $1 \mathrm{mg} / \mathrm{ml}$ of incubation media. Gas mixture of incubation flask was 95\% $0_{2}: 5 \%$ $\mathrm{CO}_{2}$. After incubation, the liver cells and bathing media are centrifuged at $25,000 \mathrm{~g}$ for $20 \mathrm{~min}$. Fibrinogen was precipitated with $25 \%$ ammonium sulphate before being converted to fibrin and measure of radio activity. Hepatocytes were obtained from liver biopsies during cholecystectomy on patients free of jaundice. The biopsy sample was immediately placed in bicarbonate buffer $\left(\mathrm{pH} 7.4,0-1^{\circ} \mathrm{C}\right)$. Cells were washed in buffer until no tricholoracetic acid inducible turbidity occurred, then weighed.

\section{Results}

Analysis of the FFA/Albumin Ratio as a Function of Thrombosis and Aging

Table 1 provides data with respect to the FFA/albumin ratio in 92 normal subjects and patients who incurred coronary thrombosis or thrombotic stroke. The ratio was found to increase in association with increase in age of the normal subject. The patient with thrombosis incurred an increase in addition to the increase imposed by aging. No significant difference appeared between data obtained in coronary thrombosis versus thrombotic stroke patients. The increase in the patient is independent of the increase associated with aging until the 60-70 year age group when magnitude of the ratio in the normal subject enters into the range associated with occurrence of a thrombotic event. Groups of patients and normal subjects matched for age show that patients exhibit significant increase above the normal subjects until reaching the 7th decade of life. Comparison of the progressive increase in the FFA/albumin ratio of the patients to the base line of normal subjects in the 20-30 year age group exhibits an increase of 35.3, $42.9,46.5$ and $47.8 \%$ respectively for the age groups of 30-40, 40-50, 50-60, and 60-70 years. The difference in the FFA/albumin ratio between normal subjects and patients declines with increasing age. By age 60-70 years, the clinically normal subjects have attained a ratio which is $34.8 \%$ greater than the ratio found for age 20-30 years. However, the 30-40 year old patient group has already attained a $35.3 \%$ increase above the ratio in the normal 20-30 year age group and displays a ratio slightly greater than the ratio found in the normal subject in the 7 th decade of life. The 60-70 year old patient shows a $47.8 \%$ increase in the ratio above that found in the normal 20-30 year group.

In summary, the FFA/albumin ratio is significantly increased in the patient with thrombosis and super imposes on the patient an increase in addition to the progressive increase occurring as a function of the aging process,
Fig. 1. By age 60-70 the clinically normal subject has incurred an increase in the ratio of $34.8 \%$ which closely approaches the $35.3 \%$ increase exhibited by the $30-40$ year age group of patients thus identifying a FFA/albumin ratio of 1.12 as a threshold associated with susceptibility to risk of thrombotic events.

Plasma Albumin Concentration as a Function of Thrombosis and Aging

The plasma concentration of albumin decreases with increasing chronological age and exhibits a further decrease in the thrombosis patient in addition to the decrease associated with aging, Fig. 2. Example is illustrated by comparing the plasma level in the 60-70 year normal group to the 30-40 year age group of thrombosis patients.

Plasma FFA Concentration as a Function of Thrombosis and Aging

The plasma level of FFA progressively increases with chronological age in the normal male Caucasian subject and exhibits an increase in the thrombosis patient in addition to the increase found in aging, Fig. 3. This change, the reverse of that observed for albumin, shows the FFA plasma level of the 30-40 and the 40-50 year old patient groups to have increased to the level observed in the 50-60 and 60-70 year age group of normal subjects.

In Vitro Induction of Increased Fibrinogen Synthesis by Hepatocytes Incubated in Patient Plasma Exhibiting Elevated FFA/Albumin Ratios

Incubation of $100 \mathrm{mg}$ of human hepatocytes for $2 \mathrm{~h}$ at $37^{\circ} \mathrm{C}$ in $2 \mathrm{ml}$ of plasma derived from patients with either coronary thrombosis or thrombotic stroke induced a significant increase in the synthesis of fibrinogen measured by incorporation of the amino acid glycine-C14 into the protein structure, Fig. 4. An average FFA/albumin molar ratio of 1.224 , found in the 60-70 year old patient group, induced a $235 \%$ increase above the FFA/albumin molar ratio of 0.828 found in the 20-30 year old group of normal subjects.

Regeneration of a Normal Rate of Fibrinogen Synthesis in Human Hepatocytes by Restoration of a Normal FFA/Albumin Molar Ratio in Plasma of Patients

Restoration of a young normal plasma albumin level in patient's plasma bearing the designated decrease in the FFA/albumin molar ratio induced a progressive decline in fibrinogen synthesis to a normal physiological rate, Fig. 4. 
Table 1 FFA/albumin ratio as a function of aging and thrombosis

\begin{tabular}{llllllll}
\hline Normal subjects & Molar ratio & Percent increase & Thrombosis patients & Molar ratio & Percent increase \\
\hline Age group & Age (years) & FFA/albumin & From age Group I & Age group & Age (years) & FFA/albumin & From age Group I \\
1 & $20-30$ & 0.828 & - & & & & \\
2 & $30-40$ & 0.900 & 8.7 & 2 & $30-40$ & 1.120 & 35.3 \\
3 & $40-50$ & 0.984 & 18.8 & 3 & $40-50$ & 1.184 & 42.9 \\
4 & $50-60$ & 1.026 & 23.9 & 4 & $50-60$ & 1.213 & 46.5 \\
5 & $60-70$ & 1.116 & 34.8 & 5 & $60-70$ & 1.224 & 47.8 \\
\hline
\end{tabular}

All values significant at $P<0.001$

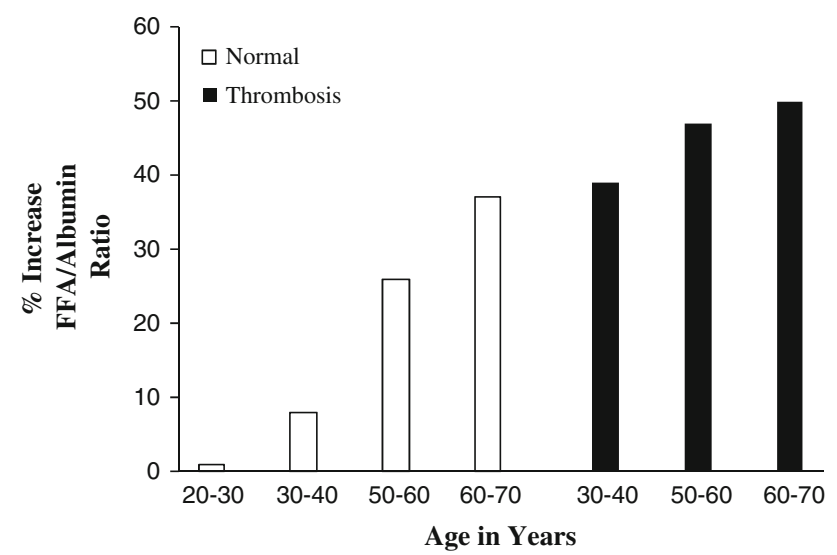

Fig. 1 FFA/albumin ratio as a function of thrombosis and aging

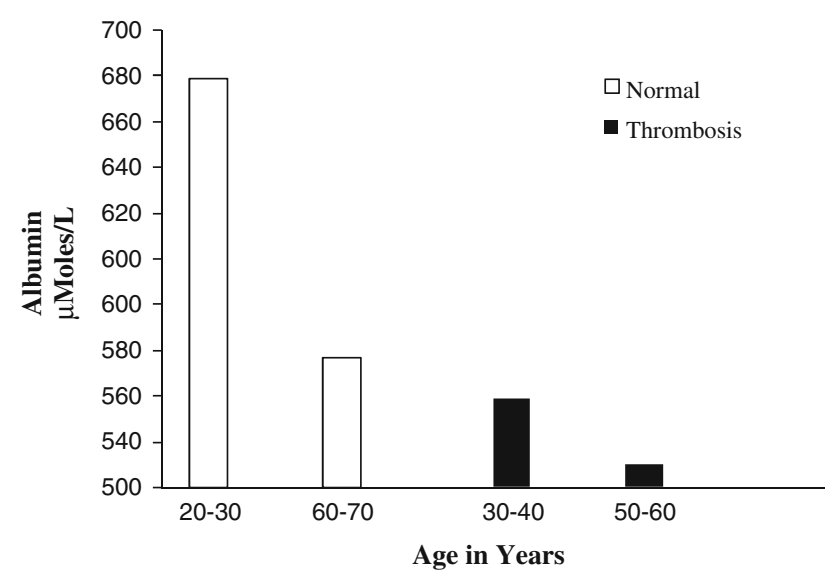

Fig. 2 Plasma albumin levels as a function of thrombosis and aging

Each bar column in Fig. 4 represents the mean of 5 separate studies with an average deviation of $3.4 \%$.

The converse of this study, based on murine hepatocytes, demonstrated that addition of Na-palmitate at a level equivalent to the maximum observed difference in the FFA level between fasted young normal male human subjects and older patients induced over a 2 fold increase in the uptake of glycine-Cl4 into fibrinogen (Pilgeram and Pickart

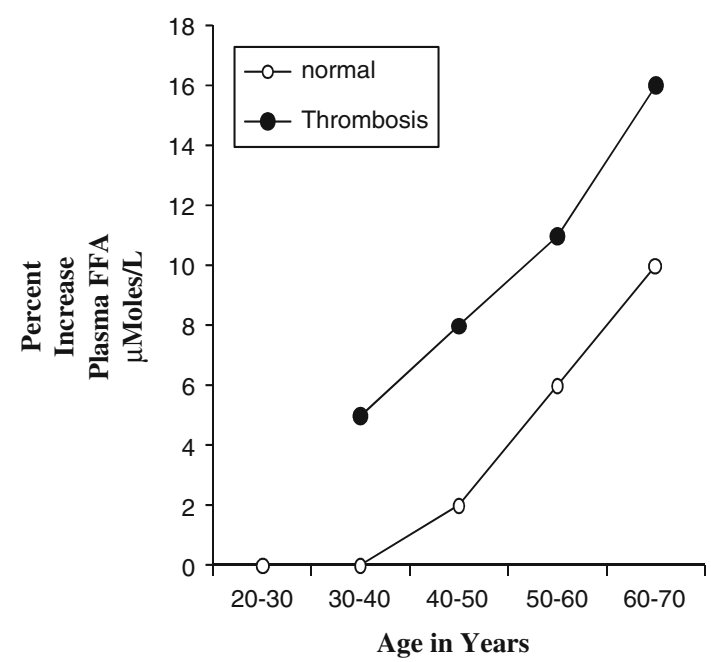

Fig. 3 Plasma FFA as a function of thrombosis and aging

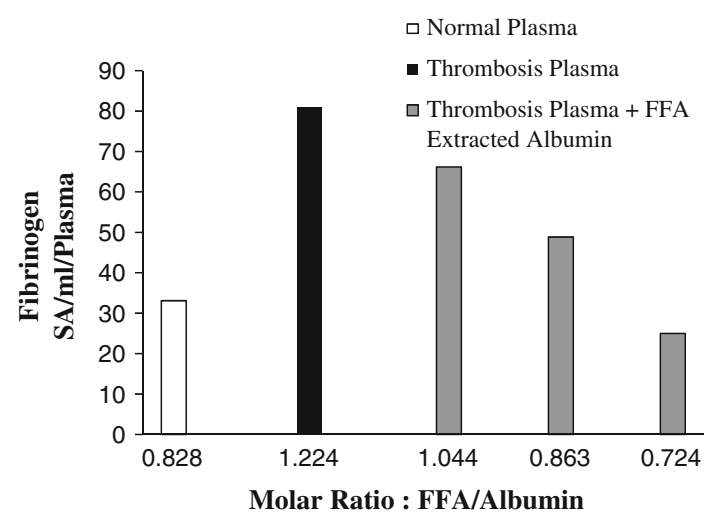

Fig. 4 Regeneration of normal fibrinogen synthesis in human hepatocytes by reduction of the FFA/albumin molar ratio in plasma of patients with thrombosis. $S A$ : specific activity fibrin (counts/min/mg)

1968). This study showed the causal factor to be specific for the FFA/albumin ratio because the plasma albumin level was maintained at a normal level in presence of increases in the ratio. The current study employed albumin as the variable controlling the ratio. 


\section{Discussion}

Biochemical interaction between albumin and fatty acids has been well documented not only by albumin serving as the obligatory cofactor for lipoprotein lipase correction of the deficiency in clearing of alimentary lipemia in plasma of patients with coronary artery disease (Pilgeram 1958; Pilgeram et al. 1964) but also by studies which demonstrated the physical chemical interaction between albumin and fatty acids including the effect of fatty acid salts and anions on albumin (Ballou et al. 1945; Goodman 1958). All of these studies document the occurrence of albumin FFA receptors which play significant roles in physiological chemical homeostasis of the living organism. The current study sought to test whether the numerical molar ratio of FFA to albumin could measure the strength of the physical chemical binding coefficient and whether this ratio served as a molecular switch or mechanism controlling the degree to which FFA controlled the rate of synthesis and turnover of fibrinogen into occlusive fibrin.

The data in this study provide evidence that the ratio serves as a measure of free fatty acid versus albumin bound fatty acid. An increasing FFA/albumin ratio measures a declining magnitude of association or binding between the protein and fatty acid. A decreasing ratio measures increasing strength of the binding coefficient or less dissociation or availability of FFA to enter into biochemical reactions independent of albumin. The differential in the binding coefficient provides the basis for the FFA/albumin ratio functioning as a mechanism or molecular switch which determines direction and magnitude of two opposing FFA biochemical pathways.

Reduction of the FFA/albumin ratio from 1.2 down to 0.8 is associated with an increased strength of the association coefficient between albumin FFA receptors and FFA which, while reducing FFA activation of fibrinogen synthesis and turnover, activates an opposing fatty acid pathway where albumin transports FFA to the mitochondrial locus of oxidative energy metabolism utilizing FFA as the substrate for generation of acetyl Co-A and oxidative energy metabolism via the citric acid cycle and oxidative phosphorylation. A beginning phase of this pathway has been described in the study where plasma albumin was discovered as the obligatory co-factor receptor of fatty acids released by the action of lipoprotein lipase in correction of the alimentary lipemia clearing deficiency found in male patients who had incurred coronary thrombosis (Pilgeram 1958; Pilgeram et al. 1964). This albumin bound FFA, labeled with $\mathrm{C} 14$, is transported to mitochondrial oxidative energy metabolism demonstrated by prompt appearance of $\mathrm{C} 14$ labeled $\mathrm{CO}_{2}$ (McCalla et al. 1957).

The function of this differential is demonstrated in the current study where downloading albumin with FFA to a ratio of 0.8 returned synthesis of fibrinogen to a normal physiological level consistent with a state of homeostasis or a steady state. Uploading albumin with FFA to a molar ratio of 1.2 weakens the binding coefficient between albumin and FFA resulting in increased levels of unbound or free fatty acids which induced increased synthesis of fibrinogen to a level found in the plasma of patients who incurred coronary thrombosis or thrombotic stroke (Pilgeram et al. 1973; Pilgeram 1973) or the development and evolution of plaques or atheroma (Duguid 1946, 1949).

FFA, dissociated from albumin binding, has been demonstrated to activate Hageman Factor (Didisheim and Mibashan 1963) which activates the cascade sequence of reactions which generate thrombin and its conversion of its natural substrate fibrinogen to the fibrin thrombus. Intra venous infusion of FFA into test animals with a vasculature free of plaques and atheroma promptly induced massive occlusive fibrin thrombi (Connor et al. 1963). In an environmental situation analogous to the human experience where an increased incidence of heart attacks occur during periods of stress, it was found that in vivo mobilization of lipid from adipose tissue fat stores generated fibrin thrombi in normal test animals (Hoak et al. 1963).

A $520 \%(P<0.001)$ increase in circulating fibrin monomers and polymers was measured in the plasma of ischemic thrombotic cerebrovascular disease (Pilgeram et al. 1973; Pilgeram 1973). These patients exhibited increased plasma levels of fibrinogen and increased generation of thrombin. Monitoring the plasma level of fibrinogen, circulating fibrin and generation of thrombin in patients who had incurred and recovered from prior ischemic events, e.g., TIA's, demonstrated progressive increase in plasma levels up to 16 days at which time clinically overt events of thrombotic icti occurred (Pilgeram 1984). The progressive level and the magnitude of this increase in the intra and extra vascular pool over a 16 day time sequence preceding each icti precluded ulceration of a plaque and procoagulant release as the cause of these thrombotic events.

It has not escaped notice that a regulatory mechanism based on a mathematical ratio between lipid and protein, which determines direction and magnitude of biochemical reactions in the systems biology of the life process, may provide insight into the nature of the highly conserved primordial evolutionary mechanism which controls aging where acceleration of the mechanism induces the major disease afflicting the human organism.

Manifestation of this control mechanism at the highest possible level of physiological or evolutionary organization based on two of the three main family groups of organic molecules, protein versus lipid, is indicative of the primacy of this code in evolutionary development of the life process and the aging mechanism. 
Acknowledgement The author expresses appreciation to Loren Pickart for expert technical assistance.

Open Access This article is distributed under the terms of the Creative Commons Attribution Noncommercial License which permits any noncommercial use, distribution, and reproduction in any medium, provided the original author(s) and source are credited.

\section{References}

Ballou G, Boyer P, Luck M. The electrophoretic mobility of human serum albumin as affected by lower fatty acid salts. J Biol Chem. 1945;159:111-6.

Benderly M, Reicher-Reiss H, Behar S, Brunner D, Goldbourt U. Fibrinogen is a predictor of mortality in coronary heart disease patients. Arterioscler Thromb Vasc Biol. 1996;16:351-6.

Burr M, Holliday R, Fehily A, Whitehead P. Haematological prognostic indices after myocardial infarction: evidence from the diet and reinfarction trial. Eur Heart J. 1992;13:166-70.

Connor W, Hoak J, Warner E. Massive thrombosis produced by fatty acid infusion. J Clin Invest. 1963;42:860-6.

Didisheim P, Mibashan R. Activation of Hageman Factor by long chain saturated fatty acids. Thromb Diath Haemorrh. 1963;9: 246-9.

Dole V, Meinertz H. Micro determination of fatty acids. J Biol Chem. 1960;235:2595-8.

Duguid JB. Thrombosis as a factor in the pathogenesis of coronary atherosclerosis. J Path Bact. 1946;58:207-17.

Duguid J. Pathogenesis of atherosclerosis. Lancet. 1949;ii:925-31.

Goodman D. The interaction of human serum albumin with long chain fatty acid anions. J Am Chem Soc. 1958;80:3892-8.

Heinrich J, Balleisen I, Schulte H, Assman G, van de Loo J. Fibrinogen and factor VII in the prediction of coronary risk. Arterioscler Thromb Vasc Biol. 1994;14:54-9.

Hoak J, Poole J, Robinson D. Thrombosis association with mobilization of fatty acids. Am J Path. 1963;43:987-98.

Kannel W, Wolf P, Castelli W, D' Agostino R. Fibrinogen and risk of cardiovascular disease. JAMA. 1987;258:1183-6.

Levenson J, Giral P, Razavian M, Gariepy J, Simon A. Fibrinogen and silent atherosclerosis in subjects with cardiovascular risk factors. Arterioscler Thromb Vasc Biol. 1995;15:1263-5.

McCalla C, Gates H, Gordon R. CO2 excretion after the intravenous administration of albumin bound palmitate-1-C14 to intact rats. Arch Biochem Biophys. 1957;71:244-53.
Meade T, Brozovic M, Chakraborti R, Haines A, North W, Sterling Y, et al. Haemostatic function and ischaemic heart disease. Principal results of the Northwick Park heart study. Lancet. 1986;ii:533-7.

Pilgeram L. Deficiencies in the lipoprotein lipase system in atherosclerosis. J Gerontol. 1958;13:32-42.

Pilgeram L. Relation of plasma fibrinogen concentration change to human arteriosclerosis. J Appl Physiol. 1961;16:660-4.

Pilgeram L. Turnover rate of autologous plasma fibrinogen-C14 in subjects with coronary thrombosis. Thromb Diath Haemorrh. 1968;20:31-43.

Pilgeram L. Detection of impending thrombosis prior to the clinically overt event. Naturwissenschaften. 1984;71:372-3.

Pilgeram L. Review: atherogenesis and fibrinogen: historical perspective and current status. Naturwissenschaften. 1993;80:547-55.

Pilgeram L. Radio isotope techniques in study of thrombosis. Symposium: IVth International Congress on Thrombosis. Wien 1973.

Pilgeram L, Pickart L. The role of thrombin in fibrinogen biosynthesis. Thromb Diath Haemorrh. 1967;17:358-64.

Pilgeram L, Pickart L. Control of fibrinogen biosynthesis; the role of free fatty acids. J Atheroscler Res. 1968;8:155-66.

Pilgeram L, Bandi Z, Thelander P. Albumin correction of the clearing factor deficiency in atherosclerosis. J Atheroscler Res. 1964;4: 244-53.

Pilgeram L, Chee A, von dem Bussehe G. Evidence for abnormalities in clotting and thrombolysis as risk factors for stroke. Stroke. 1973;4:643-57.

Schram A, Pilgeram L. Correlation of activity change in blood coagulation factors with human arteriosclerosis. Circulation. 1959;20:991.

Stone M, Thorp J. Plasma fibrinogen: a major coronary risk factor. Coll Gen Pract. 1985;35:565-9.

Toss H, Lindahl B, Siegbahn A, Wallentin I. Prognostic influence of increased fibrinogen and C-reactive protein levels in unstable coronary artery disease. Circulation. 1997;96:4204-10.

Tracy R, Bovil E, Yanez D, Psaty B, Fried I, Heiss G, et al. Fibrinogen and factor VII, but not factor VII, are associated with measures of subclinical cardiovascular disease in the elderly. Arterioscler Thromb Vasc Biol. 1995;15:1268-9.

Yarnell J, Baker I, Sweetman P, Dainton D, O'Brien J, Whitehead P, et al. Fibrinogen, viscosity and white blood cell count are major risk factors for ischemic heart disease. Circulation. 1991;83:836-44. 